\title{
Effect of Solvent on the Copolymerization of Styrene and Acrylonitrile. Application of the Bootstrap Effect to the Penultimate Unit Model
}

\author{
Bert Klumperman* and Ivo R. Kraeger \\ DSM Research, P.O. Box 18, 6160 MD Geleen, The Netherlands \\ Received August 9, 1993; Revised Manuscript Received December 13, $1993^{\circ}$
}

\begin{abstract}
The copolymerization of styrene and acrylonitrile has been investigated in bulk and in the solvents toluene, butanone, and $N, N^{\prime}$-dimethylformamide. The comparison of the copolymers prepared in the bulk system with copolymers obtained from copolymerizations in the various solvents reveals a relatively small but noticeable solvent effect. All the systems display a significant penultimate unit effect. From this work it is concluded that the copolymerization of styrene and acrylonitrile in the employed solvents can be explained by the qualitative description of the bootstrap model. The comparison of the bulk with the toluene system seems to indicate that the quantification of the bootstrap model is not realized by the assumption that the distribution coefficient $K$ is constant. A modification of the earlier proposed method for quantification of the bootstrap effect is suggested. The distribution coefficient is linearly dependent on copolymer composition. The new method provides a good fit with experimental results, whereas the used parameters have a distinct physical meaning.
\end{abstract}

\section{Introduction}

Several years ago Harwood put forward his "bootstrap model" to account for solvent effects in radical copolymerization. ${ }^{1}$ Harwood stated that copolymers having the same composition have the same sequence distribution, regardless of the solvent employed for their preparation. A growing copolymer radical seems to control its own environment during copolymerization. To explain this phenomenon Harwood proposed his bootstrap model.

The few papers published with regard to the bootstrap model only deal with qualitative aspects of the effect. ${ }^{2,3}$ Recently Klumperman and O'Driscoll presented a quantitative description of the bootstrap model. ${ }^{4}$ They proved in a mathematical way that the bootstrap model can be applied to both the terminal and the penultimate unit model and that if the bootstrap model applies, triad distribution versus copolymer composition is independent of the solvent. The authors also proposed a method for quantification of the distribution coefficient $K$ which was introduced by Harwood to describe the ratio between local and global comonomer ratio.

Four of the systems evaluated by Harwood were capable of hydrogen bonding and two of the systems yielded copolymers with Bernoullian monomer sequence distributions. Styrene and acrylonitrile are non-hydrogenbonding monomers and it is one of the major examples of a copolymerization which obeys the penultimate unit model as was pointed out, among others, by Hill et al.$^{5,6}$

Recently Tirrell and co-workers have determined the relative rates of addition of acrylonitrile and styrene to some carefully chosen low molecular radicals (model compounds) to establish the chemical feasibility of the penultimate unit effect. ${ }^{7-9}$ Their results compare favorably with those reported by Hill et al..$^{5,6}$ Harwood suggested ${ }^{1}$ that “...previously reported penultimate unit effects may be due to or at least influenced by this phenomenon...", where "this phenomenon" refers to the bootstrap effect. Klumperman and O'Driscoll ${ }^{4}$ have shown that the penultimate unit model cannot be replaced by a bootstrap effect alone. However, to study the solvent effect on a comonomer pair that has been shown to obey the pen-

- Abstract published in Advance ACS Abstracts, February 1, 1994. ultimate unit model could shed more light on the bootstrap effect in relation to this model.

When two copolymers have the same composition and monomer sequence distribution, the conditional probabilities that governed the growth of their chains must have been the same when they were formed. This phenomenon appears to be encountered when the same copolymerization is carried out in different solvents. Therefore, the conditional probabilities governing sequence distribution are unaffected by the solvent. Independence of the conditional probabilities upon solvent most probably occurs by independence of the monomer ratio at the site of propagation. A consequence of this assertion is that the real reactivity ratios are also unaffected. As a result, apparent reactivity ratios that have been determined where solvent effects were present are probably products of real reactivity ratios and distribution coefficients. The conditional probabilities are dependent on the ratio of monomer concentrations in the vicinity of the propagating radical, noted as $\left[\mathrm{M}_{1}\right] /\left[\mathrm{M}_{2}\right]$. However, this ratio of monomer concentrations need not be equal to the global monomer ratio, which is designated as $\left[\mathrm{M}_{1}{ }^{\circ}\right] /\left[\mathrm{M}_{2}{ }^{\circ}\right]$. Harwood proposed that the relationship between the two mentioned ratios may be defined as

$$
K=\frac{\left[\mathrm{M}_{1}\right] /\left[\mathrm{M}_{2}\right]}{\left[\mathrm{M}_{1}^{\circ}\right] /\left[\mathrm{M}_{2}^{\circ}\right]}
$$

If one is able to determine local monomer concentrations, real reactivity ratios can be derived. Unfortunately, an experimentally determinable coefficient of monomer partitioning $K$ is hindered by the difficulty of determining local monomer ratios.

In a recent paper, Klumperman and O'Driscoll elaborated on the quantitative aspects of the bootstrap model. 4 These authors did not focus on real reactivity ratios but stated that the only objective is to relate the apparent reactivity ratios to a reference state. Although the monomer ratio at the site of propagation in bulk copolymerization may also differ from the global monomer ratio, they defined $K$ to be unity $\left(K^{\mathrm{b}}=1\right)$ in a bulk copolymerization. It is emphasized again that bulk copolymerization is chosen as a thermodynamic reference state. This choice is arbitrary, but this is a common and 
legitimate procedure for quantification of a thermodynamic quantity. When this definition is used, it has been shown ${ }^{4}$ that the distribution coefficient $K$ is defined as

$$
K^{\mathrm{s}}=r_{i 1}^{\mathrm{B}} / r_{i 1}{ }^{\mathrm{b}}, \quad K^{\mathrm{s}}=r_{i 2}{ }^{\mathrm{b}} / r_{i 2}{ }^{\mathrm{s}}
$$

Where subscript $i$ may be 1 or 2 . The reactivity ratios $\left(r_{i j}\right)$ are defined as ratios between propagation rate constants $\left(r_{i j}=k_{i j j} / k_{i j k}\right.$, where $i, j$, and $k$ may be 1 or 2 and $\left.j \neq k\right)$. Superscriptss and b refer to solvent and bulk, respectively.

Furthermore, these authors derived equations for the transition probabilities which clarified that the relationship of sequence distribution versus copolymer composition is only dependent on $r_{\mathrm{SS}} r_{\mathrm{AA}}, r_{\mathrm{SS}} / r_{\mathrm{AS}}$, and $r_{\mathrm{AA}} / r_{\mathrm{SA}}$. Thus, the independence of triad distribution versus copolymer composition on the solvent is implied.

\section{Experimental Section}

Materials. Acrylonitrile and styrene were dried over $\mathrm{CaCl}_{2}$, distilled at reduced pressure under nitrogen at $40^{\circ} \mathrm{C}$ to remove the inhibitor, and stored at $0^{\circ} \mathrm{C}$ under nitrogen. The monomers were always used within $24 \mathrm{~h}$ after distillation. The initiator for polymerization in bulk (benzoyl peroxide) was recrystallized twice from chloroform by addition of methanol. The initiator for polymerization in solution ( $\alpha, \alpha^{\prime}$-azobis(isobutyronitrile)) was recrystallized twice from methanol. The solvents employed for polymerization were as follows: toluene (Merck), purity $>99.5 \%$, used without further purification; butanone (Riedel-de Häen), purity $99 \%$, dried over Molecular Sieves and distilled at reduced pressure under nitrogen; $N, N^{\prime}$-dimethylformamide (DMF) (Merck), purity $>99.9 \%$, used without further purification.

All other chemicals were high-purity grades and were used without purification.

Procedure. The required amounts of monomers were accurately weighed into $250.0-\mathrm{mL}$ volumetric flasks and filled with solvent (if not in bulk) to a concentration of $2.0 \mathrm{M}$ of comonomers. These mixtures were poured into $350-\mathrm{mL}$ reaction vessels and thoroughly freeze-thaw degassed (three cycles). After the last cycle the initiator was added under nitrogen $(\approx 0.1 \mathrm{~mol} \%$ with respect to the comonomers). The polymerizations were carried out under nitrogen at $60.0 \pm 0.1^{\circ} \mathrm{C}$ to conversions less than $3 \%$. For these low conversions, the copolymer formed remained soluble in the reaction mixture in all cases, except for mole fractions in the monomer feed smaller than 0.028 in styrene. The polymers were isolated by precipitation of the reaction mixture in a 10 fold excess of methanol to which $0.01 \%$ of hydroquinone was added. The conversion was determined gravimetrically. The copolymers were purified by precipitation from THF solution into methanol. Dimethyl sulfoxide (DMSO) was used as a solvent for polymers of a high acrylonitrile content. The purified polymers were dried in vacuum at $60^{\circ} \mathrm{C}$ for $36 \mathrm{~h}$.

Copolymer Compositions. The compositions of the copolymers $\left(F_{\mathrm{S}}\right.$ and $\left.F_{\mathrm{A}}\right)$ were determined (1) by duplicate percentage nitrogen analysis using a LECO FP-428 automatic elemental analyzer, (2) by calculation from the experimental triad fractions, and ( 3 ) by the relative intensities of the aromatic (S units) and methylene and methine $(A+S)$ resonances in the ${ }^{1} H$ NMR spectra. These spectra were obtained by Fourier transform techniques using, a Bruker AM-400 $(400.14 \mathrm{MHz})$ or a Varian UNITY-300 (299.95 MHz) spectrometer at $60^{\circ} \mathrm{C}$, in $5 \mathrm{~mm}$ o.d. sample tubes. Data acquisition parameters were set as follows: acquisition time, $2 \mathrm{~s}$; delay time, $10 \mathrm{~s}$; pulse width, $15 \mu \mathrm{s}$; number of transients, 16. Solutions up to $4 \%$ (w/v) in $\mathrm{CDCl}_{3}$ or DMSO$d_{6}$ were used.

Copolymer Triad Fractions. The copolymer triad fractions were obtained by Fourier transform techniques of ${ }^{13} \mathrm{C}$ NMR spectra recorded on a Varian UNITY-300 (75.43 MHz) spectrometer at $60^{\circ} \mathrm{C}$, in $10 \mathrm{~mm}$ o.d. sample tubes. Data acquisition parameters were set as follows: acquisition time, $1.5 \mathrm{~s}$; delay time, $5 \mathrm{~s}$; pulse width, $14.9 \mu \mathrm{s}$; number of transients, 4800 . Solutions of 10 to $20 \%(\mathrm{w} / \mathrm{v})$ in $\mathrm{CDCl}_{3}$ or $\mathrm{DMSO}-d_{8}$ were used. DMSO- $d_{6}$ was the solvent for copolymers that contained less than $38 \mathrm{~mol} \%$ styrene. The acrylonitrile centered and styrene centered triads have been calculated from the nitrile region (118-
Table 1. Comonomer $\left(f_{g}\right)$ and Copolymer $\left(F_{g}\right)$ Compositions Expressed as the Mole Fraction of Styrene with Conversions and Experimental Triad Fractions for the Copolymerization of Styrene and Acrylonitrile at $60^{\circ} \mathrm{C}$ in Bulk

\begin{tabular}{|c|c|c|c|c|c|c|c|c|}
\hline \multirow[b]{3}{*}{$f_{\mathrm{s}}$} & \multirow[b]{3}{*}{$\operatorname{conv}(\%)^{a}$} & \multirow[b]{3}{*}{$F_{\mathbf{s}^{b}}$} & \multicolumn{6}{|c|}{ triad fractions ${ }^{c}$} \\
\hline & & & \multicolumn{3}{|c|}{ S-centered } & \multicolumn{3}{|c|}{ A-centered } \\
\hline & & & SSS & $\begin{array}{l}\text { SSA } \\
\text { ASS }\end{array}$ & ASA & AAA & $\begin{array}{l}\text { AAS } \\
\text { SAA }\end{array}$ & SAS \\
\hline 0.0151 & 1.5 & 0.138 & 0.00 & 0.00 & 1.00 & $0.74^{*}$ & $0.24^{*}$ & $0.02 *$ \\
\hline 0.0155 & 1.3 & 0.147 & 0.00 & 0.00 & 1.00 & $0.75^{*}$ & $0.23^{*}$ & $0.02 *$ \\
\hline 0.0531 & 2.0 & 0.318 & 0.00 & 0.06 & 0.94 & 0.24 & 0.62 & 0.14 \\
\hline 0.1037 & 2.5 & 0.393 & 0.02 & 0.14 & 0.85 & 0.11 & 0.56 & 0.33 \\
\hline 0.1095 & 3.1 & 0.371 & 0.00 & 0.09 & 0.91 & 0.13 & 0.57 & 0.30 \\
\hline 0.2214 & 2.5 & 0.466 & 0.02 & 0.27 & 0.71 & 0.05 & 0.39 & 0.56 \\
\hline 0.586 & 1.8 & 0.598 & 0.13 & 0.55 & 0.32 & 0.00 & 0.13 & 0.87 \\
\hline 0.6198 & 2.8 & 0.632 & 0.18 & 0.54 & 0.28 & 0.00 & 0.12 & 0.88 \\
\hline 0.800 & 1.2 & 0.693 & 0.29 & 0.58 & 0.13 & 0.00 & 0.08 & 0.92 \\
\hline 0.939 & 1.2 & 0.832 & 0.62 & 0.34 & 0.04 & 0.00 & 0.05 & 0.95 \\
\hline
\end{tabular}

a Weight percent of total monomer feed. ${ }^{b}$ Arithmetic average from $\% \mathrm{~N}$ elemental analysis, ${ }^{1} \mathrm{H}-\mathrm{NMR}$ spectra, and from the triad fractions; estimated error $F_{\mathrm{S}} \neq 1.0 \% .^{\circ}$ From ${ }^{13} \mathrm{C}$ NMR spectra; estimated absolute error in triad fraction \pm 0.03 .

Table 2. Comonomer $\left(f_{g}\right)$ and Copolymer $\left(F_{g}\right)$ Compositions Expressed as the Mole Fraction of Styrene with Conversions and Experimental Triad Fractions for the Copolymerization of Styrene and Acrylonitrile at $60^{\circ} \mathrm{C}$ in Toluene

\begin{tabular}{|c|c|c|c|c|c|c|c|c|}
\hline \multirow[b]{3}{*}{$f_{\mathrm{s}}$} & \multirow[b]{3}{*}{$\operatorname{conv}(\%)^{a}$} & \multirow[b]{3}{*}{$F_{\mathbf{S}^{b}}^{b}$} & \multicolumn{6}{|c|}{ triad fractiong ${ }^{c}$} \\
\hline & & & \multicolumn{3}{|c|}{ S-centered } & \multicolumn{3}{|c|}{ A-centered } \\
\hline & & & SSS & $\begin{array}{c}\text { SSA } \\
\text { ASS }\end{array}$ & ASA & AAA & $\begin{array}{l}\text { AAS } \\
\text { SAA }\end{array}$ & SAS \\
\hline 0.0151 & 1.7 & 0.092 & 0.00 & 0.00 & 1.00 & $0.84 *$ & $0.15^{*}$ & $0.01^{*}$ \\
\hline 0.0152 & 1.3 & 0.092 & 0.00 & 0.03 & 0.97 & $0.83^{*}$ & $0.16^{*}$ & $0.01 *$ \\
\hline 0.0377 & 1.1 & 0.233 & 0.00 & 0.03 & 0.97 & 0.52 & 0.45 & 0.03 \\
\hline 0.1101 & 1.8 & 0.366 & 0.00 & 0.09 & 0.91 & 0.16 & 0.57 & 0.27 \\
\hline 0.1110 & 1.8 & 0.361 & 0.00 & 0.08 & 0.92 & 0.19 & 0.57 & 0.24 \\
\hline 0.1899 & 1.9 & 0.434 & 0.01 & 0.23 & 0.76 & 0.07 & 0.46 & 0.47 \\
\hline 0.3241 & 2.3 & 0.496 & 0.03 & 0.35 & 0.62 & 0.03 & 0.31 & 0.66 \\
\hline 0.5009 & 2.6 & 0.566 & 0.07 & 0.50 & 0.43 & 0.01 & 0.18 & 0.81 \\
\hline 0.5727 & 2.1 & 0.591 & 0.11 & 0.53 & 0.36 & 0.00 & 0.15 & 0.85 \\
\hline 0.5763 & 2.6 & 0.594 & 0.10 & 0.55 & 0.35 & 0.01 & 0.14 & 0.85 \\
\hline 0.7555 & 1.9 & 0.670 & 0.24 & 0.58 & 0.18 & 0.00 & 0.08 & 0.92 \\
\hline 0.9334 & 1.4 & 0.818 & 0.61 & 0.36 & 0.03 & 0.00 & 0.03 & 0.97 \\
\hline 0.9349 & 1.4 & 0.827 & 0.62 & 0.36 & 0.03 & 0.00 & 0.03 & 0.97 \\
\hline
\end{tabular}

${ }^{a}$ Weight percent of total monomer feed. ${ }^{b}$ Arithmetic average from $\% \mathrm{~N}$ elemental analysis, ${ }^{1} \mathrm{H}-\mathrm{NMR}$ spectra, and from the triad fractions; estimated error $F_{\mathrm{S}} \pm 1.0 \% .{ }^{\mathrm{c}}$ From ${ }^{13} \mathrm{C}$ NMR spectra; estimated absolute error in triad fraction \pm 0.03 .

$123 \mathrm{ppm}$ ) and the quaternary aromatic region (139-145 ppm), respectively. Spectral assignments were carried out according to previously published chemical shifts. ${ }^{10}$ For the high acrylonitrile content measurements (measured in DMSO- $d_{6}$ ) a correction was made for overlap between the AAA and AAS + SAA triads. The appropriate correction factor was determined from the tacticity-dependent signals of the acrylonitrile methine resonances at $27.7,27.2$, and $26.6 \mathrm{ppm}(\mathrm{rr}, \mathrm{rm}$, and $\mathrm{mm}$, respectively). The data to which this correction method was applied are indicated with an asterisk in the Tables 1-4.

\section{Results and Discussion}

Copolymerizations of styrene and acrylonitrile have been conducted in bulk, toluene, butanone, and DMF. In Tables 1-4 the copolymer compositions, expressed as the mole fractions of styrene, for the studied systems are outlined together with the triad fraction data. The results for the copolymer compositions represent arithmetic averages of ${ }^{1} \mathrm{H} \mathrm{NMR},{ }^{13} \mathrm{C} \mathrm{NMR}$, and percent nitrogen analysis.

All the systems display a significant penultimate unit effect, which is recognized from the reactivity ratios listed 
Table 3. Comonomer $\left(f_{\mathrm{g}}\right)$ and Copolymer $\left(F_{\mathrm{g}}\right)$ Compositions Expressed as the Mole Fraction of Styrene with Conversions and Experimental Triad Fractions for the Copolymerization of Styrene and Acrylonitrile at $60^{\circ} \mathrm{C}$ in Butanone

\begin{tabular}{|c|c|c|c|c|c|c|c|c|}
\hline \multirow[b]{3}{*}{$f_{\mathrm{s}}$} & \multirow[b]{3}{*}{$\operatorname{conv}(\%)^{a}$} & \multirow[b]{3}{*}{$F_{\mathrm{S}}^{b}$} & \multicolumn{6}{|c|}{ triad fractions $^{c}$} \\
\hline & & & \multicolumn{3}{|c|}{ S-centered } & \multicolumn{3}{|c|}{ A-centered } \\
\hline & & & SSS & $\begin{array}{l}\text { SSA } \\
\text { ASS }\end{array}$ & ASA & AAA & $\begin{array}{l}\text { AAS } \\
\text { SAA }\end{array}$ & SAS \\
\hline 0.0147 & 0.7 & 0.145 & 0.00 & 0.05 & 0.95 & $0.69 *$ & $0.29 *$ & $0.02^{*}$ \\
\hline & & 135 & 0.02 & 0.01 & 0.97 & $0.71 *$ & $0.28^{*}$ & $0.01 *$ \\
\hline 0.0279 & 2.8 & 0.220 & 0.00 & 0.03 & 0.97 & 0.45 & 0.50 & 0.05 \\
\hline 0.0281 & 4.2 & 0.222 & 0.00 & 0.03 & 0.97 & 0.44 & 0.51 & 0.05 \\
\hline 0.1092 & 2.6 & 0.374 & 0.00 & 0.10 & 0.90 & 0.16 & 0.58 & 0.26 \\
\hline 0.1093 & 2.4 & 0.377 & 0.00 & 0.11 & 0.89 & 0.14 & 0.58 & 0.28 \\
\hline 0.4010 & & 0.527 & 0.05 & 0.43 & 0.52 & 0.01 & 0.27 & 0.72 \\
\hline & 2.3 & $0.5 \varsigma$ & 0.11 & 0.55 & 0.34 & 0.00 & 0.16 & 0.84 \\
\hline & 3.9 & 0.73 & 0.39 & 0.51 & 0.10 & 0.00 & 0.05 & 0.95 \\
\hline & 3.0 & 0.732 & 0.39 & 0.52 & 0.09 & 0.00 & 0.05 & 0.95 \\
\hline & 1.9 & 0.745 & 0.42 & 0.50 & 0.08 & 0.00 & 0.05 & 0.95 \\
\hline & 1.2 & 0.768 & 0.48 & 0.46 & 0.06 & 0.00 & 0.05 & 0.95 \\
\hline & 1.2 & & 0.47 & 0.46 & 0.06 & 0.00 & 0.04 & 0.96 \\
\hline & 1.0 & 0.830 & 0.65 & 0.32 & 0.03 & 0.01 & 0.03 & 0.96 \\
\hline
\end{tabular}

a Weight percent of total monomer feed. ${ }^{b}$ Arithmetic average from $\% \mathrm{~N}$ elemental analysis, ${ }^{1} \mathrm{H}-\mathrm{NMR}$ spectra, and from the triad fractions; estimated error $F_{S} \pm 1.0 \% \cdot{ }^{c}$ From ${ }^{13} \mathrm{C}$ NMR spectra; estimated absolute error in triad fraction \pm 0.03 .

Table 4. Comonomer $\left(f_{g}\right)$ and Copolymer $\left(F_{g}\right)$ Compositiong Expressed as the Mole Fraction of Styrene with Conversions and Experimental Triad Fractions for the Copolymerization of Styrene and Acrylonitrile at $60^{\circ} \mathrm{C}$ in DMF

\begin{tabular}{|c|c|c|c|c|c|c|c|c|}
\hline \multirow[b]{3}{*}{$f \mathrm{~s}$} & \multirow[b]{3}{*}{$\operatorname{conv}(\%)^{a}$} & \multirow[b]{3}{*}{$F_{\mathrm{s}}^{b}$} & \multicolumn{6}{|c|}{ triad fractionge } \\
\hline & & & \multicolumn{3}{|c|}{ S-centered } & \multicolumn{3}{|c|}{ A-centered } \\
\hline & & & SSS & $\begin{array}{l}\text { SSA } \\
\text { ASS }\end{array}$ & ASA & AAA & $\begin{array}{l}\text { AAS } \\
\text { SAA }\end{array}$ & SAS \\
\hline 0.0149 & 4.4 & 0.101 & 0.00 & 0.04 & 0.96 & $0.81^{*}$ & $0.18^{*}$ & $0.01 *$ \\
\hline 0.0150 & 6.3 & 0.094 & 0.00 & 0.02 & 0.98 & $0.84^{*}$ & $0.15^{*}$ & $0.01 *$ \\
\hline 0.0151 & 2.5 & 0.113 & 0.00 & 0.03 & 0.97 & $0.83^{*}$ & $0.16^{*}$ & $0.01^{*}$ \\
\hline 0.0406 & 2.8 & 0.231 & 0.00 & 0.03 & 0.97 & 0.45 & 0.50 & 0.05 \\
\hline 0.1084 & 4.8 & 0.336 & 0.00 & 0.08 & 0.92 & 0.22 & 0.59 & 0.19 \\
\hline 0.1085 & 2.5 & 0.337 & 0.00 & 0.08 & 0.92 & 0.21 & 0.59 & 0.20 \\
\hline 0.1091 & 2.4 & 0.333 & 0.00 & 0.07 & 0.93 & 0.22 & 0.57 & 0.21 \\
\hline 0.2639 & 2.2 & 0.452 & 0.02 & 0.27 & 0.71 & 0.07 & 0.44 & 0.49 \\
\hline 0.4182 & 3.0 & 0.495 & 0.04 & 0.40 & 0.56 & 0.03 & 0.31 & 0.66 \\
\hline 0.5765 & 2.7 & 0.556 & 0.09 & 0.51 & 0.40 & 0.01 & 0.21 & 0.78 \\
\hline 0.5769 & 2.9 & 0.587 & 0.10 & 0.52 & 0.38 & 0.00 & 0.20 & 0.80 \\
\hline 0.7503 & 2.7 & 0.596 & 0.20 & 0.56 & 0.24 & 0.00 & 0.12 & 0.88 \\
\hline 0.8 & 1.2 & 0.698 & 0.33 & 0.54 & 0.13 & 0.00 & 0.07 & 0.93 \\
\hline & 1.0 & 0.788 & 0.62 & 0.35 & 0.03 & 0.00 & 0.03 & 0.97 \\
\hline & 0.7 & 0.821 & 0.64 & 0.34 & 0.02 & 0.00 & 0.03 & 0.97 \\
\hline
\end{tabular}

- Weight percent of total monomer feed. ${ }^{b}$ Arithmetic average from $\% \mathrm{~N}$ elemental analysis, ${ }^{1} \mathrm{H}-\mathrm{NMR}$ spectra, and from the triad fractions; estimated error $F_{\mathrm{s}} \pm 1.0 \% .^{\mathrm{c}}$ From ${ }^{13} \mathrm{C}$ NMR spectra; estimated absolute error in triad fraction \pm 0.03 .

Table 5. Reactivity Ratios with Standard Deviations Calculated for the Penultimate Unit Models from the NLLS Fit to the Copolymer Composition Data and Triad Fraction Data for the Copolymerization of Styrene and Acrylonitrile in Bulk, Toluene, Butanone, and DMF at $60^{\circ} \mathrm{C}$

\begin{tabular}{lcccc}
\hline solvent & \multicolumn{1}{c}{$r_{\mathrm{SS}}$} & \multicolumn{1}{c}{$r_{\mathrm{AS}}$} & \multicolumn{1}{c}{$r_{\mathrm{SA}}$} & \multicolumn{1}{c}{$r_{\mathrm{AA}}$} \\
\hline bulk & $0.250 \pm 0.013$ & $0.604 \pm 0.028$ & $0.105 \pm 0.007$ & $0.070 \pm 0.004$ \\
toluene & $0.242 \pm 0.008$ & $0.559 \pm 0.016$ & $0.119 \pm 0.005$ & $0.108 \pm 0.005$ \\
butanone & $0.284 \pm 0.005$ & $0.570 \pm 0.017$ & $0.132 \pm 0.005$ & $0.063 \pm 0.002$ \\
DMF & $0.227 \pm 0.011$ & $0.440 \pm 0.020$ & $0.175 \pm 0.009$ & $0.105 \pm 0.005$ \\
bulk, acc. & 0.229 & 0.634 & 0.091 & 0.039 \\
to Hill & & & &
\end{tabular}

in Table 5. The reactivity ratios were determined according to a NLLS technique based on principals outlined by Britt and Luecke. ${ }^{11}$ In the NLLS optimization tech-

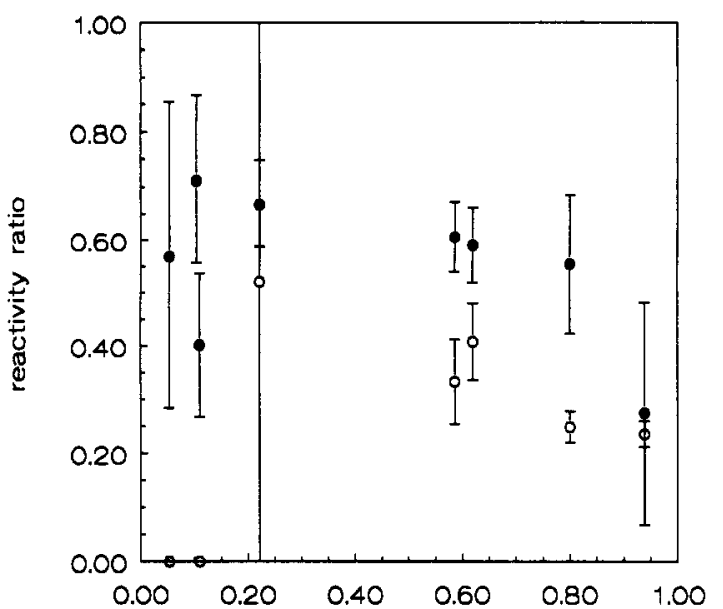

fs

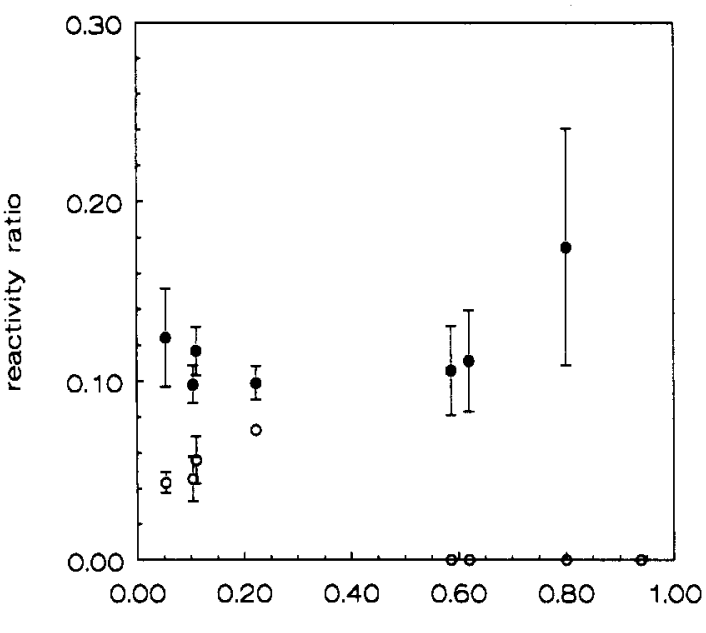

fs

Figure 1. Calculated reactivity ratios as a function of styrene fraction in the monomer feed for the copolymerization of styrene and acrylonitrile in bulk at $60^{\circ} \mathrm{C}$, using Chajb's equations:12 (top) $0=r_{\mathrm{SS}}$ and $0=r_{\mathrm{AS}}$ (bottom) $0=r_{\mathrm{AA}}$ and $0=r_{\mathrm{SA}}$.

nique, a set of seven equations was used to determine reactivity ratios. One equation describes copolymer composition as a function of monomer feed. The other six equations describe each of the styrene and acrylonitrile centered triad fractions as a function of monomer feed. The sums of squares of all seven equations are minimized simultaneously.

The penultimate unit effect is also confirmed by means of Châjô's equations, ${ }^{12}$ although to our opinion this method is somewhat less suitable for this system. Due to the large areas where either the fraction SSS or the fraction AAA is approximately zero (resp. $F_{\mathrm{S}}<0.45$ and $F_{\mathrm{S}}>0.55$ ), large errors are found in the reactivity ratios calculated from these equations, as can be seen from the error bars in Figure 1. Thus, conclusions with respect to the occurrence of a penultimate unit effect can only be based on a limited region of monomer feed ratios. Qualitatively, the results compare favorably with those obtained by Hill et $a .^{3}$

The relationship of copolymer composition versus monomer feed composition becomes more clear in Figure 2 where the copolymer composition curves for the different systems are compared. With concern to the copolymer composition curve in Figure 2, the toluene system resembles the bulk system in the high styrene region $\left(f_{s}>0.5\right)$. The copolymer composition curves of the bulk and the toluene system do not coincide in the high acrylonitrile region $\left(f_{\mathrm{S}}<0.5\right)$. 


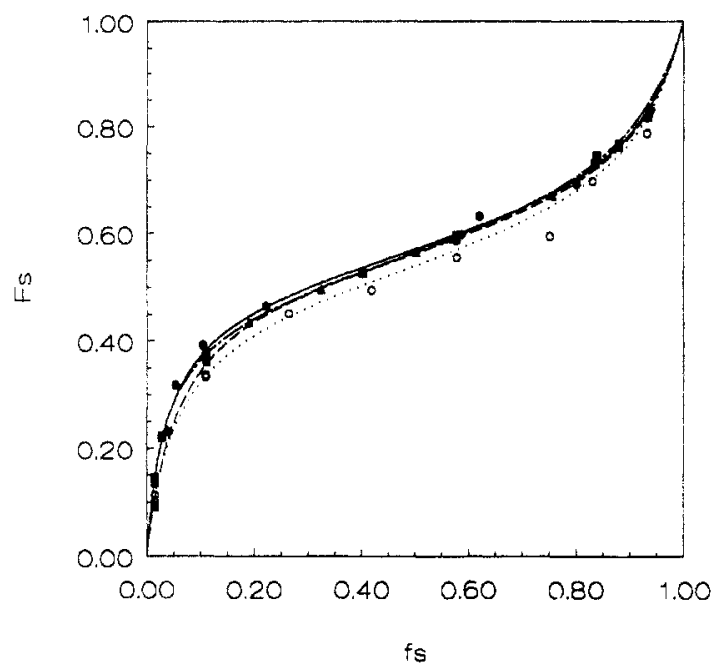

Figure 2. Copolymer composition curves for the copolymerizations of styrene and acrylonitrile in bulk, toluene, butanone, and DMF at $60^{\circ} \mathrm{C} . F_{\mathrm{S}}$ is the mole fraction of styrene in the copolymer and $f_{s}$ is the mole fraction of styrene in the comonomer feed. $($ drawn $)=$ bulk; $\Delta$ (dashed $)=$ toluene; $\square($ dash-dot $)=$ butanone; $O$ (dotted) = DMF. Curves are calculated using the reactivity ratios given in Table 5 .

In toluene the polarity of the solvent and the styrenerich SAN is comparable. Hill et al..$^{6}$ also investigated the SAN copolymerization in acetonitrile. They noticed that the copolymer composition curves of the bulk and the acetonitrile system coincided in the high acrylonitrile region $\left(f_{\mathrm{s}}<0.3\right)$. In acetonitrile the polarity of the solvent is comparable with the acrylonitrile-rich SAN.

The copolymerizations run in DMF might demonstrate the same trend, when polarity is concerned. However, from Figure 2 it is obvious that the copolymer composition curve for polymerizations in DMF is not in agreement with this proposition. DMF and acetonitrile are comparable if solubility parameters are concerned $\left(24.8 \times 10^{3}\right.$ and $24.3 \times 10^{3}\left[\mathrm{~J} / \mathrm{m}^{3}\right]^{1 / 2}$, respectively). ${ }^{13}$ The difference in this case may probably arise from subtle differences in interactions between electron donor and acceptor molecules. It is known that styrene acts as an electron donor whereas acrylonitrile is an electron acceptor. Effects of solvents on this type of interaction have been reported in literature, e.g. in a recent review by Rätzsch and Vogl. ${ }^{14}$ It is hypothesized that donor-acceptor interactions will also play a significant role in the bootstrap effect.

The copolymer composition versus monomer feed curve for the polymerizations in butanone resembles the bulk system over the entire monomer feed region. Probably due to small differences in polarity, no significant deviation in apparent reactivity ratios is observed between butanone and bulk.

From Figure 3 it is recognized qualitatively that for the copolymerization of SAN in bulk and in the solvents toluene, butanone, and DMF the bootstrap effect is important; i.e. the relationship of monomer sequence distribution versus copolymer composition is independent upon solvent.

However, from Figure 2 it is recognized that the copolymer composition curves for the polymerizations in bulk and in toluene only deviate in the high acrylonitrile region. In this region the dominating parameter is $r_{\mathrm{AA}}$. The value of $r_{\mathrm{AA}}$ is the only reactivity ratio that seems to deviate when the values determined in bulk and in toluene are compared with the $r_{\mathrm{AA}}$ values of the other systems.

If a solvent has an effect on a copolymerization and for instance changes $r_{\mathrm{AA}}$, the quantitative description of the bootstrap theory implies that the solvent should affect
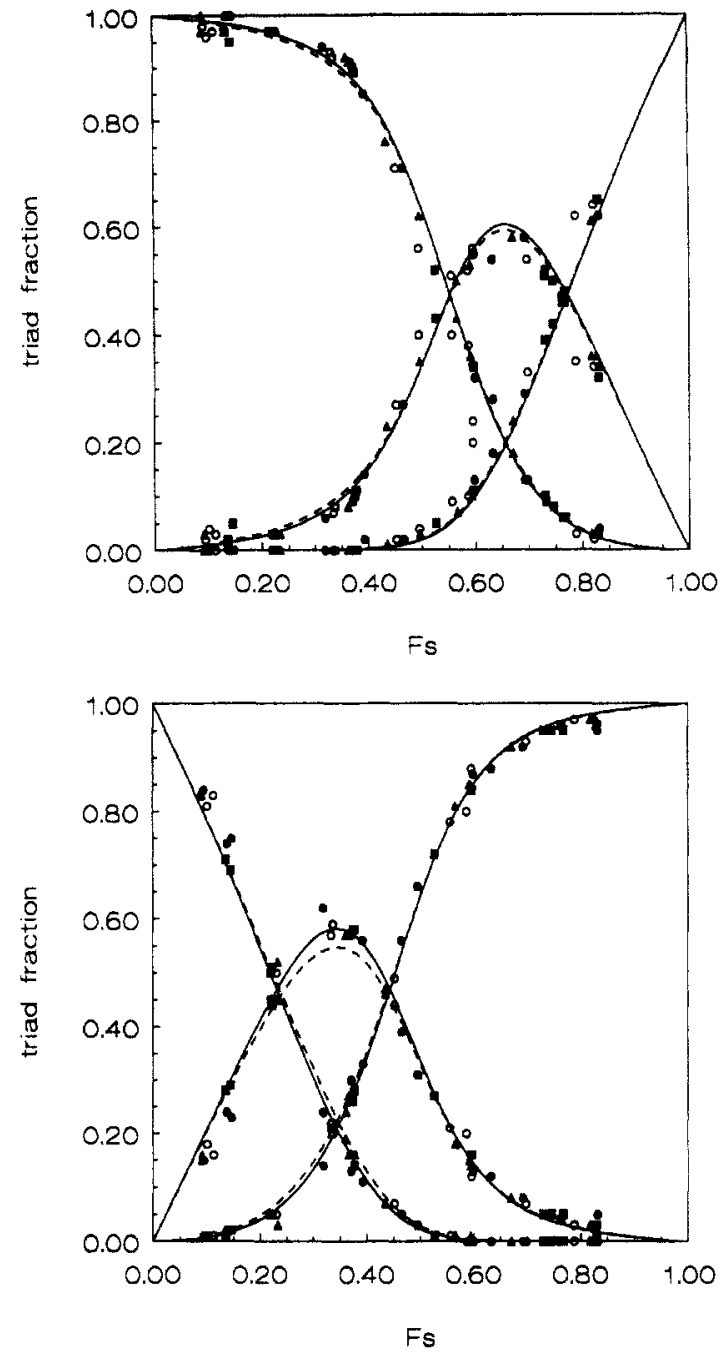

Figure 3. Comparison between the triad fractions for SAN copolymers prepared in bulk $(\Theta)$, toluene $(\Delta)$, butanone $(\mathbb{D})$, and DMF (O) at $60^{\circ} \mathrm{C}$. The styrene-centered (top), and acrylonitrilecentered (bottom) triad fractions have been plotted against the copolymer composition, $F_{8}$. Dashed curves are calculated using average values of the reactivity ratios from Table 5 . Drawn curves are calculated using the reactivity ratios from Table 7.

Table 6. Distribution Coefficient $\boldsymbol{K}$ Calculated According to Equation 2 with Standard Deviationge for the

Copolymerization of Styrene and Acrylonitrile in Toluene, Butanone, and DMF at $60^{\circ} \mathrm{C}$

\begin{tabular}{lcccc}
\hline solvent & $K$ (from $\left.r_{\mathrm{SS}}\right)$ & $K$ (from $\left.r_{\mathrm{AS}}\right)$ & $K$ (from $\left.r_{\mathrm{SA}}\right)$ & $K$ (from $r_{\mathrm{AN}}$ ) \\
\hline toluene & $0.97 \pm 0.06$ & $0.93 \pm 0.05$ & $0.88 \pm 0.08$ & $0.65 \pm 0.07$ \\
butanone & $1.14 \pm 0.05$ & $0.94 \pm 0.06$ & $0.80 \pm 0.08$ & $1.11 \pm 0.07$ \\
DMF & $0.91 \pm 0.07$ & $0.73 \pm 0.06$ & $0.60 \pm 0.08$ & $0.67 \pm 0.07$
\end{tabular}

a Standard deviations calculated according to the equation $\Delta K=$ $K\left[\left(\Delta r_{i j} / r_{i j}\right)^{2}+\left(\Delta r_{i j}^{b} / r_{i j}^{b}\right)^{2}\right]^{1 / 2}$, where $i$ and $j$ may be 1 or 2 .

the other reactivity ratios to the same extent so as to keep the earlier mentioned reactivity ratio products and quotients constant. For the apparent reactivity ratios from Table 5 this is not the case. This is easily recognized from the values of the distribution coefficients from Table 6 which are calculated from the different reactivity ratios as indicated, according to eq 2 . The distribution coefficients $K$ depend on the reactivity ratio from which they are determined. For example, the values of $K$ determined from $r_{\text {SS }}$ and from $r_{\mathrm{AA}}$ are in bad agreement for copolymerizations in toluene and DMF. The cause for this phenomenon may be found in an assumption which is made in the method of quantification, described in an earlier publication. ${ }^{4}$ 
The distribution coefficient $K$ describes the partitioning of the monomers between free solvent phase and locus of polymerization (the polymer coil). In the proposed method for quantification, this distribution coefficient is implicitly assumed to be independent of copolymer composition. This assumption may induce an error, whose magnitude is governed by the difference in polarity among both the comonomers and the solvent. For example, imagine a hypothetical system consisting of a solvent of moderate polarity and two comonomers, one of which has a high polarity and the other having a low polarity. By going from the homopolymer of the first monomer gradually to the homopolymer of the second, the solvent phase first is the phase with the lower polarity and eventually becomes the phase with the higher polarity. The distribution coefficient in this system would change from a certain value below unity to a value above unity (or vice versa, depending on the definition). At the initial definition of the bootstrap effect and the distribution coefficient, ${ }^{1}$ it was already stated that $K$ will be influenced by the composition of the growing polymer chain.

A clear indication for a changing distribution coefficient is provided by work of Hill et al..$^{3}$ and from this work. The copolymerization of STY and ACN in bulk, toluene, and acetonitrile clearly shows this effect. If the copolymerization in bulk is compared with those in toluene and acetonitrile with respect to copolymer composition versus monomer feed, it is found that in a certain range of monomer feed ratios the curves coincide and in another range they do not. Coinciding curves mean, in terms of the bootstrap effect, that the distribution coefficients are equal (unity in case of bulk copolymerization). Based on these observations a change in the quantification of the bootstrap effect is proposed. If the bootstrap effect is determined by differences in polarity between solvent and polymer phase, a simple relationship could be used to relate the distribution coefficient to copolymer composition. The polarity of the copolymer phase is directly related to the copolymer composition. The polarity of the solvent is constant. The distribution coefficient could therefore be expressed as

$$
K=A F_{1}+B
$$

Then for each comonomer pair one set of reactivity ratios describes the reference state (bulk copolymerization) and for each solvent a value for $A$ and $B$ is determined to describe the partitioning between solvent and copolymer coil. The physical meaning of the parameters $A$ and $B$ is then comparable to that of the distribution coefficient $K$. That is, $B$ is the distribution coefficient for the homopolymer of monomer 2 , while $A+B$ is that of the homopolymer of monomer 1.

Physically more correct would be to define a copolymer composition dependent distribution coefficient for bulk copolymerization, since it is not the solvent but the copolymer that causes the deviation from constancy. However, it is again for the sake of quantification that the distribution coefficient for bulk copolymerization is defined to be unity. From this reference state the $A$ and $B$ values are determined with a meaning as outlined above.

In this work we analyzed the experimental data using the above proposed technique by a NLLS fitting procedure. ${ }^{11}$ We used data for bulk and three solvents (toluene, butanone, and DMF) to determine the best values for the reactivity ratios and three sets of $A$ and $B$ values, corresponding with the solvents. The data for bulk and the three solvents were fitted simultaneously, so as to get the most reliable values for the reactivity ratios. Monomer
Table 7. Reactivity Ratios and Distribution Coefficients $(K)$ for the Copolymerization of Styrene and Acrylonitrile in Bulk, Toluene, Butanone, and DMF at $60^{\circ} \mathrm{C}$ '

\begin{tabular}{lccccccc}
\hline \multicolumn{1}{c}{ solvent } & $r_{\mathrm{SS}}$ & $r_{\mathrm{AS}}$ & $r_{\mathrm{SA}}$ & $r_{\mathrm{AA}}$ & $A$ & $B$ & $A+B$ \\
\hline bulk & 0.249 & 0.581 & 0.122 & 0.063 & $0^{b}$ & $1^{b}$ & $1^{b}$ \\
toluene & 0.249 & 0.581 & 0.122 & 0.063 & -0.25 & 1.11 & 0.86 \\
butanone & 0.249 & 0.581 & 0.122 & 0.063 & -0.04 & 1.07 & 1.03 \\
DMF & 0.249 & 0.581 & 0.122 & 0.063 & 0.18 & 0.22 & 0.40
\end{tabular}

${ }^{a} K$ is defined as $K=A F_{1}+B \cdot{ }^{b}$ By definition $K=1$ for bulk copolymerization and therefore, $A=0$ and $B=1$.

feed, copolymer composition, and STY and ACN centered triad fractions were used in the parameter estimation program. In the parameter estimation procedure a correction was made for conversion by a numerical technique to average the calculated values over a given conversion interval. The results of the parameter estimation are listed in Table 7.

In Table 7, the values for $B$, which equal the distribution coefficients for poly $(\mathrm{ACN})\left(K_{\mathrm{ACN}}\right)$, and the values for $A$ $+B$, which equal the distribution coefficient for poly(STY) $\left(K_{\mathrm{STY}}\right)$, are listed. There appears to be a strong difference between DMF on one hand and toluene and butanone on the other. The latter two have distribution coefficients that are approximately unity over the entire copolymer composition range.

The trends for these two solvents over the composition range are limited. Especially for butanone there is no significant influence of the copolymer composition on the distribution coefficient. For toluene the vicinity of the growing polymer chain becomes relatively richer in styrene when the copolymer becomes richer in acrylonitrile (i.e. $A<0$ ), although the effect is rather limited. This phenomenon may be induced by specific interactions between monomer and polymer (i.e. monomeric styrene having a stronger affinity toward poly(ACN) and/or monomeric acrylonitrile having a stronger affinity toward poly(STY)). However, one should bear in mind that the distribution coefficients listed in Table 7 are relative to bulk copolymerization. Thus, no strong conclusions can be based on the observed trend in the case of toluene.

For DMF the distribution coefficient is below unity over the entire copolymer composition range. This means that the vicinity of the growing polymer chain is relatively poor in styrene. Apparently, the polymer chain is preferentially surrounded by acrylonitrile to a larger extent than in the case of bulk copolymerization. The effect is larger for acrylonitrile-rich copolymers than for styrene-rich copolymers (i.e. $A>0$ ).

This observation is opposite to the general tendency that the use of nonpolar solvent favors the incorporation of the polar monomer. ${ }^{1}$ Again this may be due to donoracceptor interactions, rather than ordinary polarity effects. Measurements and/or calculations with respect to preferential solvation or any type of complex formation should shed more light on the origin of these observations in the future.

As an example of the difference between classical reactivity ratios and the present approach, the experimentally determined triad distributions versus monomer feed compositions in toluene are given in Figure 4 with the calculated curves according to the reactivity ratios from Table 5 and according to the reactivity ratios and $A$ and $B$ values from Table 7 .

The latter curves are obtained by an iterative process, since apparent reactivity ratios depend on copolymer composition (through $K$ ). In this iterative process copolymer composition and triad distribution are calculated by using the reactivity ratios for the reference state (bulk). 

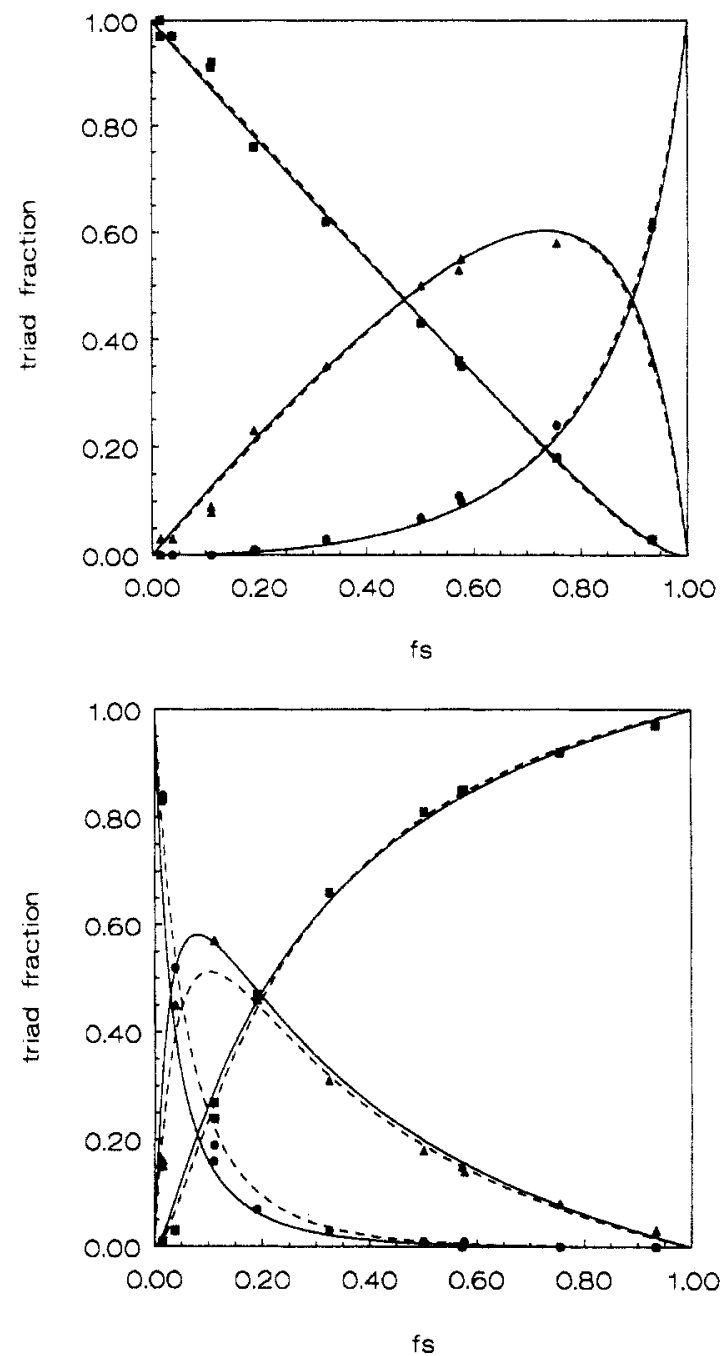

Figure 4. Experimentally determined triad fractions versus monomer feed composition for the SAN copolymerization in toluene at $60^{\circ} \mathrm{C}$. The styrene-centered (top) and acrylonitrilecentered (bottom) triad fractions have been plotted, together with curves calculated using the reactivity ratios from Table 5 (dashed curves) and using reactivity ratios and distribution coefficient from Table 7 (drawn curves).

Based on these copolymer compositions the corresponding apparent reactivity ratios are calculated by invoking the $A$ and $B$ values for toluene.

Copolymer composition and triad distribution are recalculated and new apparent reactivity ratios are determined. This procedure is repeated until the calculated copolymer composition and triad distribution converge.

\section{Conclusions}

Similar to previous studies, in this study the existence of a penultimate unit effect is clearly demonstrated. This is easily recognized from the calculated reactivity ratios. In our opinion, the use of Chajô's equations is for this particular copolymerization somewhat less suitable. This is due to the limited regions of monomer feed ratios where the penultimate unit effect causes significant differences within the relevant reactivity ratio pairs (i.e. $r_{11}$ compared with $r_{21}$ and $r_{22}$ compared with $r_{12}$ ).
The occurrence of the bootstrap effect is demonstrated from the solvent independence of monomer sequence distribution versus copolymer composition. For this comonomer pair, the evidence is not strong, since the solvent effect is limited and the used data (triad fractions based on ${ }^{13} \mathrm{C}$ NMR measurements) contain a rather large uncertainty. Nevertheless, the solvent effects have been analyzed in terms of the bootstrap effect. In this analysis it was clear that quantification of the bootstrap effect as presented before and tested on STY/MMA and STY/ MAnh data did not provide an adequate description of this system.

The implicit assumption in the previously reported method that the distribution coefficient $(K)$ is independent on copolymer composition gives rise to an inconsistency of $K$ values derived from the different reactivity ratios. From these experiments it is clear that $K$ is dependent on copolymer composition. Based on the physical background of the distribution coefficient this can be easily understood.

The new approach which provides an adequate description of the experimental results has the following characteristics:

Each comonomer pair is described by one set of reactivity ratios (we use bulk copolymerization as the reference state).

For each solvent employed, the solvent effect is described by two parameters. These parameters seem to account for the difference in polarity between the solvent and each of the two monomers.

Acknowledgment. We thank people from the Analytical Chemistry Department of DSM Research for analyzing the copolymer samples (J. Beulen and G. Kolfschoten, NMR; F. Gilissen, elemental analysis). Furthermore, we thank T. Tiemersma and M. Zilverstand for their assistance in the NLLS-fitting procedures and Professor K. O'Driscoll for helpful discussions. The permission of DSM Research to publish this work is gratefully acknowledged.

\section{References and Notes}

(1) Harwood, H. J. Makromol. Chem., Macromol. Symp. 1987, 10 / 11, 331-354.

(2) Davis, T. P. Polym. Commun. 1990, 31, 442-444.

(3) Hill, D. J. T.; Lang, A. P.; Munro, P. D.; O'Donnell, J. H. Eur. Polym. J. 1992, 28, 391-398.

(4) Klumperman, B.; O'Driscoll, K. F. Polymer 1993, 34, 10321037.

(5) Hill,D.J.T.; O'Donnell, J.H.; O'Sullivan, P.W.Macromolecules 1982, 15, 960-966.

(6) Hill, D. J. T.; Lang, A. P.; O’Donnell, J. H. Eur. Polym. J. 1991, $27,765-772$.

(7) Jones, S. A.; Prementine, G. S.; Tirrell, D. A. J. Am. Chem. Soc. 1985, 107, 5275-5276.

(8) Cywar, D. A.; Tirrell, D. A. Macromolecules 1986, 19, 29082911.

(9) Prementine, G. S.; Tirrell, D. A. Macromolecules 1987, 20, 30343038.

(10) Pham, Q. T.; et al. Proton and Carbon NMR Spectra of Polymers, 1st ed.; Penton Press: London, 1991.

(11) Britt, H. I.; Luecke, R. H. Technometrics 1973, 15, 233-247.

(12) Chajo, R.; Ubara, H.; Nishioka, A. Polym.J. 1972, 3, 670-674.

(13) Polymer Handbook, 2nd ed.; Brandrup, J., Immergut, E. H., Eds.; 1975.

(14) Rätzsch, M.; Vogl, O. Prog. Polym. Sci. 1991, 16, 279-301. 